\title{
Developing test methods for interfacial ageing in composite insulators
}

DOI:

10.1109/CEIDP.2015.7352083

Link to publication record in Manchester Research Explorer

\section{Citation for published version (APA):}

Bastidas, P. D., \& Rowland, S. M. (2015). Developing test methods for interfacial ageing in composite insulators. In host publication (pp. 294-297). IEEE. https://doi.org/10.1109/CEIDP.2015.7352083

\section{Published in:}

host publication

\section{Citing this paper}

Please note that where the full-text provided on Manchester Research Explorer is the Author Accepted Manuscript or Proof version this may differ from the final Published version. If citing, it is advised that you check and use the publisher's definitive version.

\section{General rights}

Copyright and moral rights for the publications made accessible in the Research Explorer are retained by the authors and/or other copyright owners and it is a condition of accessing publications that users recognise and abide by the legal requirements associated with these rights.

\section{Takedown policy}

If you believe that this document breaches copyright please refer to the University of Manchester's Takedown Procedures [http://man.ac.uk/04Y6Bo] or contact uml.scholarlycommunications@manchester.ac.uk providing relevant details, so we can investigate your claim.

\section{OPEN ACCESS}


This is the accepted manuscript, which has been accepted by IEEE for publication (C) 2015 . Personal use of this material is permitted. Permission from IEEE must be obtained for all other uses, in any current or future media, including reprinting/republishing this material for advertising or promotional purposes, creating new collective works, for resale or redistribution to servers or lists, or reuse of any copyrighted component of this work in other works. The full reference is:

\section{'Developing test methods for interfacial ageing in composite insulators'}

P. D. Bastidas and S. M. Rowland

IEEE Conference on Electrical Insulation and Dielectric Phenomena,

pp. 594-597, 2015

DOI: $\underline{10.1109 / C E I D P .2015 .7352083}$ 


\title{
Developing test methods for interfacial ageing in composite insulators
}

\author{
Pablo D. Bastidas and Simon M. Rowland \\ School of Electrical and Electronic Engineering \\ The University of Manchester \\ Manchester, United Kingdom \\ s.rowland@manchester.ac.uk
}

\begin{abstract}
High voltage insulation systems consist of a combination of different insulating materials, exposed to multiple stresses during operation. Outdoor composite insulators used in transmission lines are typically made of two solid polymeric materials bonded together to form the insulator. Work reported here contributes to the evaluation of degradation within internal interfaces by developing tests to investigate interfacial tracking, with a view to replicating the tracking phenomena seen within composite insulators in service. The present paper highlights high voltage tests on dielectric interfaces with needle electrodes generating local high fields to initiate tracking. FEA has been used to design an experiment with controlled electric field magnitudes over a $1 \mathrm{~m}$ sample. Moreover, information from monitoring partial discharges and surface temperature is used to understand the inception and propagation of tracking activity within the structure.
\end{abstract}

Keywords - interface; interfacial; partial discharges; tracking; composite insulator; needle; FEA

\section{INTRODUCTION}

High voltage outdoor composite insulators are produced from glass-fibre reinforced polymer for the high strength core, and are jacketed with carefully selected polymers, such as silicon rubber $(\mathrm{SiR})$, for external protection. The interfaces between these materials are generally parallel to high electric fields, and within these interfaces points of concentrated electrical stress or electrical weakness can produce locations of discharge activity. These points may take the form of conducting foreign bodies or cavities, for example. Surface tracking is the formation and growth of conducting carbonaceous paths over an insulating material under the action of electrical discharges, and has been studied for many years [1]. Failure due to tracking along interfaces is also well known in faulty composite insulators [2], however standardised tests do not exist for interfacial tracking: and this paper contributes to the development of such tests.

\section{PREVIOUS TEST REGIMES}

Interfacial degradation is more difficult to study than surface ageing because it is not visible from the outside of the equipment unless in a very advanced state. Also the control and characterisation of an interface is more difficult than for a surface. Visibility to the eye normally only results after development of interfacial tracking through the jacket of the insulator to the outside surface of the housing [3]. If defects are

The authors are grateful to EPSRC and The University of Manchester for a DTA grant in support of this project. present, internal tracking can occur because the resins used in the glass-fibre composite form carbon deposits in the presence of discharges. On the other hand, materials are chosen for the sheath which do not form carbon in the presence of discharges because it is accepted that discharges will occur on the surface at some time in the insulators life. However these latter materials such as SiR can erode deeply to puncture the sheath, resulting in moisture ingress to the core [2]. It is clear there are many factors which can influence the internal degradation processes. Gubanski et al. report both the development of ageing tests and diagnostic tools to assess the quality of interfaces in composite insulators [4]. Key factors include: the internal pressure on the interface, surface roughness, the presence of greases, the electric field distribution [5],[6]. Kutil and Froshlic [7] have reported that poor bonding can lead to a complete breakdown along the interface, due to delaminated areas and micro-cracks in the medium.

There have been several approaches to studying interfacial degradation of polymers proposing methods to analyse the interfacial breakdown. For instance, research findings by Takahashi et al. [8] presented two types of system to explore interfacial breakdown at the interface between $\mathrm{SiR}$ and epoxy resin. The first system allows investigation of interfacial discharge inception between solids, whilst the second system simulates delamination. However, no evidence of the internal degradation is presented. The importance of the primer bonding at the interface between SiR and epoxy, focusing on the interfacial mechanical strengths between these two polymers has been studied in [9], an electrode wire of $75 \mu \mathrm{m}$ radius was used to provide field enhancement. The experimental results show different effects on SiR and epoxy. The SiR samples presented white powder on the surface but less damage in the bulk, whereas the epoxy produced surface cracks.

Several studies have used needles as electrodes between two insulating materials in small scale samples. For example, Dang and Fournier [10] analysed the dielectric performance of interfaces using new and field-aged cable joints as samples, with EPDM/EPDM and EPDM/XLPE interfaces. Two needles were used as electrodes, separated by $4 \mathrm{~mm}$ from each other, the main feature being the effect of loss of interfacial pressure on the dielectric strength of the samples, whilst the breakdown voltage at the interfaces are recorded.

Another example of studying interfaces for cable joint applications by $\mathrm{Du}$ and $\mathrm{Gu}$ [11] also analysed the interfacial tracking failure at a SiR and XLPE interface using a needle- 
plane configuration separated by $10 \mathrm{~mm}$. The study was aimed at investigating the effect of different interfacial pressures on the distribution of carbonisation patterns and discharge light. This study confirmed that increased pressure reduces internal discharges intensity and tracking propagation at the interfaces.

Kamer and Ieda [12] developed an experiment involving inserting a needle into an interface to produce high local electrical stress, to create tracking within the interface, as Fig. 1 shows. In this test, the result is visible tracking development of the effect of introducing the needle at the interface. This paper presents a broad view of the issues of interfacial tracking in its widest sense, and is particularly focused on bushing applications.

Bolliger and Boggs [13] used planar point-to-edge electrodes separated by $10 \mathrm{~mm}$, to evaluate the chemistry of interfacial tracking, using different combinations of materials. The data obtained show that the heat from electrical discharges facilitates the formation of hydrocarbons from free radicals formed at these interfaces, playing an important role in the chemistry as initiators of degradation of the dielectrics. It is suggested that high interfacial pressure and the use of grease at the interface are good techniques to avoid interfacial breakdown.

In a different approach to test exposed interfaces, Andersson et al. [14] combined the salt-fog and the inclinedplane test. In this they tested end-on joints between polymer materials. They proposed this method to test mechanical strength at interfaces between samples by adding/curing interfaces against glued interfaces.

As the above review illustrates, the study of interfacial tracking is mainly uses small scale samples. The problem of these small scale tests is that the front edge of the track sees an increased field as the track grows and reduces the gap to the opposite electrode. This does not represent the conditions of track growth in overhead line insulators in practice, and will fundamentally change the rate of propagation. Therefore, this project presents the use of large test samples to investigate interfacial tracking in more realistic conditions. Extensive work using FEA has generated an experimental design that closely controls the field in which the defect grows. By using large scale experiments and good field control, the advancing tip of a track does not experience a significant change in field as it grows - replicating service conditions. In this study we have replicated the most common practice of using metallic needles to generate high, divergent fields to initiate tracks.

\section{EXPERIMENTAL}

The aim of the work presented is to establish an experimental facility which enables the study of tracking at the interface of polymeric materials. To complement the traditional small, flat samples described in the literature, here a full-scale long-rod experimental model is used as seen in Fig. 2. The stress

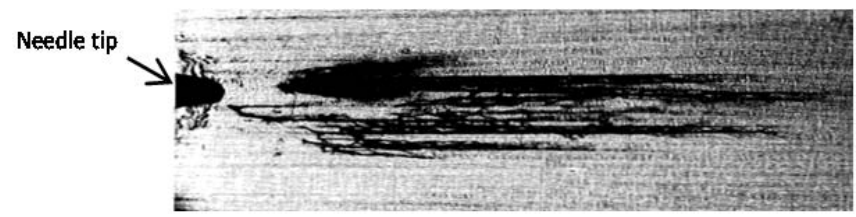

Figure 1. Interfacial treeing/tracking in fibre-glass reinforced epoxy [12] controlling corona rings were designed using FEA to ensure a uniform field in the absence of defects. The FEA model is shown in Fig. 3. In this paper all voltages and fields reported are AC peak values, unless otherwise stated.

In the following experiments, transparent heat-shrink tubing and unfilled epoxy resin have been applied to core rods as disparate sheathing materials. A hollow tube of white PVC has been used as the core, because this makes it easy to identify tracking activity: PVC tracks readily leaving a distinct black track in contrast to the original white surface. It is not expected that the tracking characteristics will be the same as commercial core resins, but it is ideal for developing the experimental protocols. In these cases tracking can be seen through the sheath materials.

In this experimental structure of a sheath over a hollow rod, we have in effect created a layered structure of two planes with no edges. Tracking at the interface between the two material will continue until the track can emerge onto one of their surfaces.

Fig. 4 shows the experimental circuit which consists of a $170 \mathrm{kV} \mathrm{rms} \mathrm{AC} 50 \mathrm{~Hz}$ transformer, and a $10 \mathrm{k} \Omega$ ballast resistor which is used to protect the circuit from high current. The high voltage is measured on an oscilloscope with a 1000:1 voltage divider. The coupling capacitor is connected with the Omicron

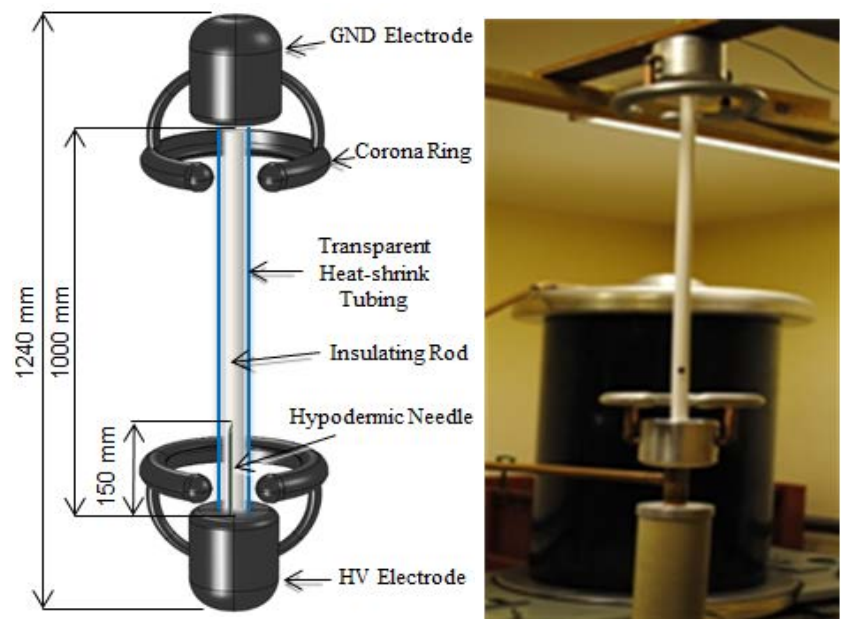

Figure 2. Schematic and photograph of the long-rod experiment

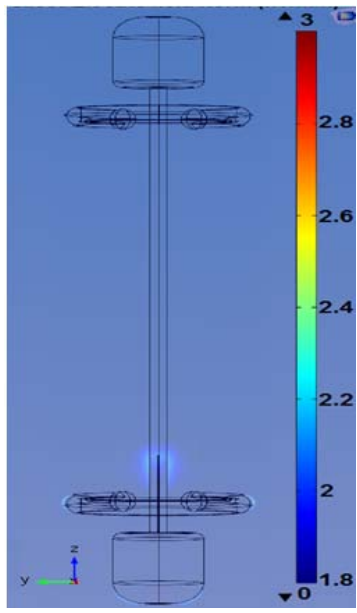

Figure 3. 3D FEA model of the long-rod experiment, in this case incorporating a conducting track at the $\mathrm{HV}$ end. The electric field values on the guide are in $\mathrm{kV} / \mathrm{mm}$. 
M600 PD measurement system to determine the partial discharge (PD) magnitudes, and the partial discharge inception voltage (PDIV). Infrared (IR) thermal imaging is used to establish a relationship between the surface temperature and PD from the electrical discharges at the interface.

Conductive defects were placed at the interface between two types of polymeric materials to initiate interfacial tracking. Introducing a hypodermic needle at the sheath-core interface had the effect of enhancing the electric field magnitude, and creating a high divergent field, producing partial discharges capable of degrading the insulating material, and so initiating interfacial tracking [5],[12].

\section{RESULTS AND DISCUSSION}

The experiments in the long-rod system examined three key design issues. Firstly, the relationship between the sheath outer temperature and the characteristics of the interfacial discharges was investigated. Secondly, the development of conductive carbon at the core-to-sheath interface was studied. Thirdly, the surface temperature required for a track to pierce the insulator sheath was considered.

a) Puncture of the Sheathing: Table I displays the nature of failure of the sheathing under $\mathrm{HV}$ as the sheath thickness and material was changed. It was observed that a thin polyethylene sheath $(<2 \mathrm{~mm}$ thick) would be easily pierced due to the internal discharges, because of its low thermal mass and resistance.

A thicker sheath of epoxy resin was more robust than the PVC core tube so that a $5 \mathrm{~mm}$ thick sheath withstood the heat longer than the PVC core, so that the hollow tube was punctured before the sheath, as expected. Clearly this process of internal puncture could not occur when a solid rod core is used, but provides an alternative sample structure for future use and study of epoxy resins. The internal surface of the PVC rod after puncture is shown in Fig. 5.

Fig. 6 shows the relationship between maximum PD magnitude and the surface temperature of a $5 \mathrm{~mm}$ thick epoxy sheath. It can be seen that PD magnitude increases with voltage as expected, and that as a result, surface temperature also increases with voltage.

As seen in Fig. 6, with a $5 \mathrm{~mm}$ epoxy sheath at $195 \mathrm{kV}$, the PVC rod was pierced, as a result of tracking which developed on the rod's inner surface. Although the external temperature was only $41^{\circ} \mathrm{C}$ the temperature of the thinner PVC core was certainly higher: It is a disadvantage of this system that the PVC surface temperature cannot be directly measured. The sample prepared with $3 \mathrm{~mm}$ thick epoxy sheath developed interfacial tracking which will be discussed in more detail in the next section.

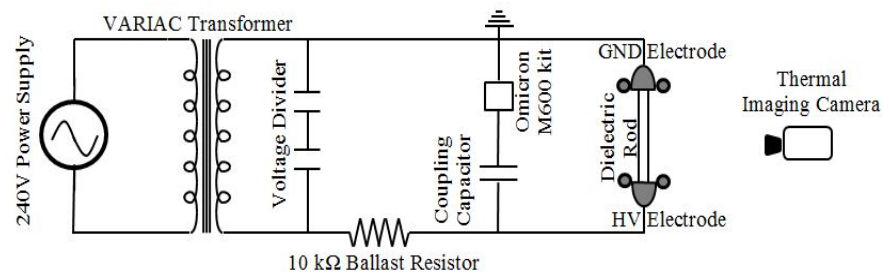

Figure 4. Experimental circuit
TABLE I. FAILURE MECHANISM OF SAMPLES

\begin{tabular}{cc}
\hline Sheath & Type of Degradation \\
\hline $1.9 \mathrm{~mm}$ PE heat shrink & Punctured the PE layer \\
$3 \mathrm{~mm}$ thick epoxy & Interfacial tracking \\
$5 \mathrm{~mm}$ thick epoxy & Punctured the PVC rod \\
\hline
\end{tabular}

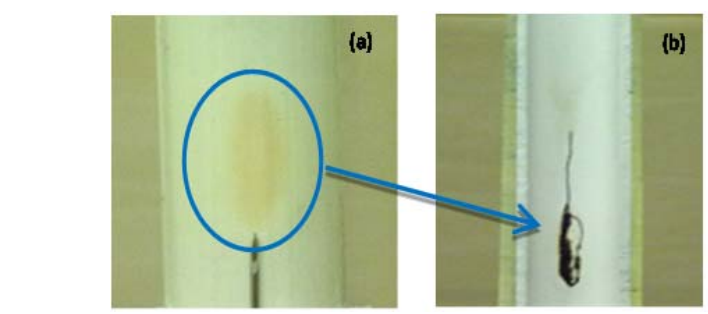

Figure 5. (a) Tracking development on the PVC tube's inner surface from the tip of a hypodermic needle

(b) Magnified view of a carbon path of $36 \mathrm{~mm}$ length

Final observations whilst carrying out these experiments are as follows:

- This experimental arrangement allowed development of interfacial tracking.

- PD could be monitored and was seen to be strongly voltage dependent

- Surface temperature of the outer sheath only could be monitored and increased with PD activity.

- The transition from tracking at the interface to puncturing the sheath and surface tracking can be reproduced.

- The epoxy layer can be readily changed in this arrangement, effectively allowing what is normally the core material to be readily studied.

b) Tracking at Interfaces: In the second part of the long-rod experiment, a hypodermic needle was embedded between the PVC rod and epoxy resin sheath. A partial discharge inception voltage (PDIV) of $50 \mathrm{kV}$ was observed. This value is heavily dependent on the volume around the needle point and whether it sits in a void. Carbon paint was also used as a contaminant

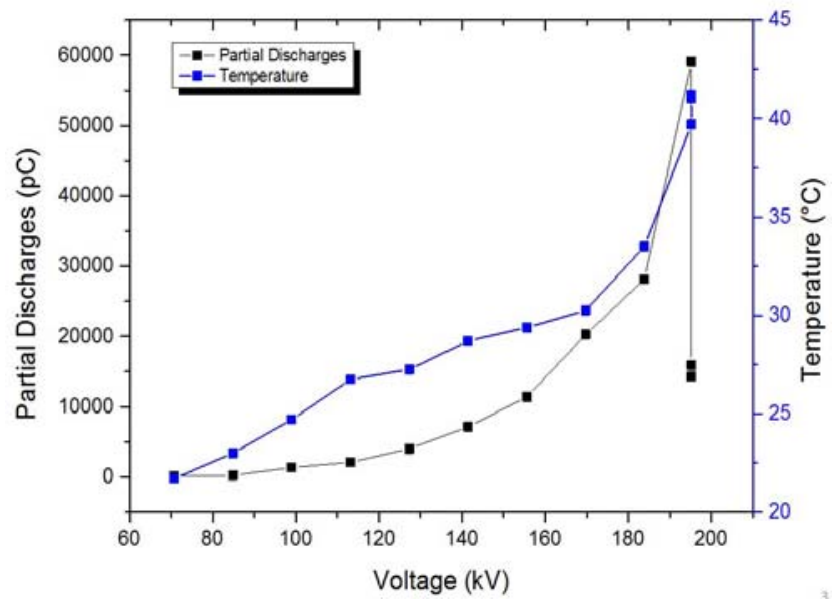

Figure 6. Relationship between applied voltage (kV peak), temperature $\left({ }^{\circ} \mathrm{C}\right)$ and PD (pC) with a $5 \mathrm{~mm}$ sheath 


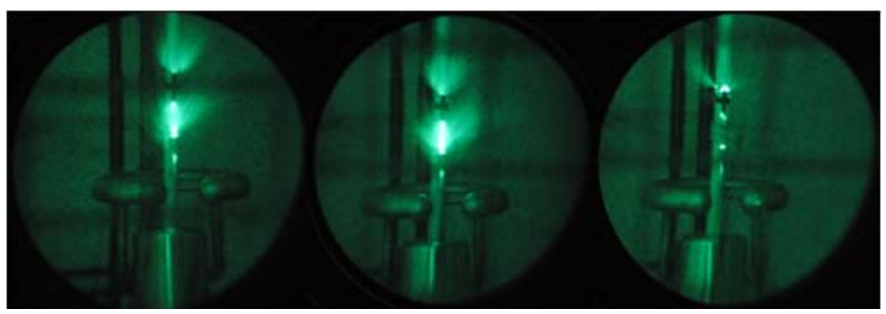

(a) $80 \mathrm{kV}$

(b) $130 \mathrm{kV}$

(c) $210 \mathrm{kV}$

Figure 7. UV imaging of corona in a sample containing a carbon track

on the PVC rod to encourage the track propagation at the $\mathrm{rod} / \mathrm{sheath}$ interface. Fig. 7 shows the development of an experiment in which a $37 \mathrm{~mm}$ long carbon track was painted on the interface before assembly using aqueous carbon solution, $22 \mathrm{~mm}$ distant from an $\mathrm{HV}$ hypodermic needle. Above $80 \mathrm{kV}$ the corona intensity increases at the extreme ends of the painted carbon track and the hypodermic needle as expected. This experiment was run at $210 \mathrm{kV}$, at which voltage the PD magnitudes ranged between of 40 to $60 \mathrm{nC}$ when the interfacial tracking was developing. After a period ( $\sim 20$ mins) the carbon path was extended in length by interfacial tracking, bridging the gap between the hypodermic needle and the painted carbon track. At this point, PD magnitudes up to $70 \mathrm{nC}$ were recorded. Thereafter, the PD's and the noise were reduced significantly to $10 \mathrm{nC}$.

Fig. 8 shows the resulting carbon path of $22 \mathrm{~mm}$ length at the interface of the two polymer materials.

\section{CONCLUSION}

The experimental work presented here has developed a long-rod sample for studying tracking at the interface of two polymers. This preliminary work suggests that it is possible to create and monitor the growth of tracking at the interfaces using large reproducible samples of a geometry similar to overhead line insulators. Moreover, from experimental testing, it was observed that the development of conductive interface tracking within interfaces has four stages. These are:

- Internal conductive defects enhance the local electric field resulting in partial discharge activity.

- Conductive carbon deposits form as a result of high energy electron discharges.

- The resulting degradation extends to from carbonaceous conductive paths. These will further enhance the electric field intensity, causing the continued growth of the internal track.

- Intense discharges at the leading edge of the track elevate the temperature of the local materials. If this temperature is excessive the track comes to the outer surface of the sheath and flashover results.

The long-rod hollow sample developed, allows the epoxy layer to be on the surface of the sample, providing additional flexibility in studying both the sheath and core materials.

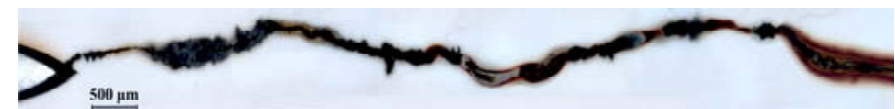

Figure 8. Magnified view of a carbon path of $22 \mathrm{~mm}$ length formed by discharges at the interface between the two polymers

\section{REFERENCES}

[1] M. Billings, A. Smith, and R. Wilkins, "Tracking in Polymeric Insulation," IEEE Trans. Electr. Insul., vol. EI-2, no. 3, pp. 131-137, Dec. 1967.

[2] F. Schmuck, I. Gutman, N. Mahatho, M. Perez, A. Phillips, A. Pigini, G. Pirovano, J. Seifert, M. R. Shariati, V. Sklenicka, W. Vosloo, and R. Wesley, "Assessment of in-service composite insulators by using diagnostic tools," GIGRE WG B2.21, no. August, pp. 1-71, 2013.

[3] A. J. Carreira, E. A. Cherney, R. A. Christman, E. Cleckley, J. Kuffel, A. J. Phillips, and J. Varner, "Guidelines for establishing diagnostic procedures for live-line working of nonceramic insulators," IEEE Trans. Power Deliv., vol. 29, no. 1, pp. 126-130, 2014.

[4] S. M. Gubanski, A. Dernfalk, J. Andersson, and H. Hillborg, "Diagnostic methods for outdoor polymeric insulators," IEEE Transactions on Dielectrics and Electrical Insulation, vol. 14. pp. 1065-1080, 2007.

[5] R. Ross, "Dealing with interface problems in polymer cable terminations," IEEE Electrical Insulation Magazine VOL. 15, No. 4, vol. 15 , no. 4, pp. 5-9, 1999.

[6] H. Geene, R. Ross, D. Kunze, M. Nagao, F. Ombello, W. Strassberger, J. Cardinaels, J. Gahungu, J. Kim, J. Densley, and T. Blackburn, "Interfaces in accessories for extruded HV and EHV cables," CIGRE Joint Task Force 21/15, pp. 52-59, 2002.

[7] A. Kutil and K. Froshlich, "Partial discharge phenomena in composite insulation materials," Annual Report of the Conference on Electrical Insulation and Dielectric Phenomena. pp. 343-347, 1995.

[8] T. Takahashi, T. Okamoto, Y. Ohki, and K. Shibata, "Breakdown strength at the interface between epoxy resin and silicone rubber-a basic study for the development of all solid insulation," IEEE Trans. Dielectr. Electr. Insul., vol. 12, no. 4, 2005.

[9] J. Andersson, S. M. Gubanski, and H. Hillborg, "Properties of interfaces between silicone rubber and epoxy," IEEE Trans. Dielectr. Electr. Insul., vol. 15, no. 5, pp. 1360-1367, Oct. 2008.

[10] C. Dang and D. Fournier, "Dielectric performance of interfaces in premolded cable joints," IEEE Trans. Power Deliv., vol. 12, no. 1, pp. 29-32, 1997.

[11] B. X. Du and L. Gu, "Effects of interfacial pressure on tracking failure between XLPE and silicon rubber," IEEE Trans. Dielectr. Electr. Insul., vol. 17, no. 6, pp. 1922-1930, Dec. 2010.

[12] H. Kamer and M. Ieda, "Technical aspects of interfacial phenomena in solid insulating systems," 3rd Int. Conf. Prop. Appl. Dielectr. Mater., pp. 592-597, 1991.

[13] D. A. Bolliger and S. A. Boggs, "The chemistry of interfacial tracking," IEEE Trans. Dielectr. Electr. Insul., vol. 19, no. 3, pp. 996-1006, Jun. 2012.

[14] J. Andersson, S. Gubanski, and H. Hillborg, "Properties of interfaces in silicone rubber," IEEE Trans. Dielectr. Electr. Insul., vol. 14, no. 1, pp. 137-145, Feb. 2007. 